\title{
AVALIAÇÃO DA FERTILIDADE DOS SOLOS DE SISTEMAS AGROFLORESTAIS COM CAFEEIRO (Coffea arabica L.) EM LAVRAS-MG ${ }^{1}$
}

Bruno Grandi Salgado², Renato Luiz Grisi Macedo², Maria Inês Nogueira Alvarenga ${ }^{3}$ Nelson Venturin ${ }^{2}$

\begin{abstract}
RESUMO - Com o objetivo de avaliar a fertilidade dos solos em um sistema agroflorestal composto por cafeeiros (Coffea arábica L. - Mundo Novo), ingazeiros (Inga vera Willd) e grevíleas (Grevilea robusta A. Cunn), situado em Lavras, Minas Gerais, foi instalado o presente experimento. Usou-se um delineamento experimental inteiramente casualizado com três tratamentos e sete repetições. Os tratamentos foram: a) cafeeiros a pleno sol, b) cafeeiros consorciados com ingazeiros e c) cafeeiros consociados com grevílea. Os espaçamentos dos cafeeiros nos três sistemas foi 4 x $1 \mathrm{~m}$, para o ingazeiro $8 \mathrm{~m}$ x $15 \mathrm{~m}$ e para a grevílea $12 \mathrm{~m} \mathrm{x} 10 \mathrm{~m}$. Aos 15 anos de idade do cafeeiro e do ingazeiro e aos 9 anos da grevílea foram avaliadas as seguintes características dos solos, $\mathrm{pH}$, acidez potencial $\left(\mathrm{H}^{+} \mathrm{Al}\right)$, alumínio trocável $\left(\mathrm{Al}^{+3}\right)$, bases trocáveis $\left(\mathrm{Ca}^{+2} \mathrm{e} \mathrm{Mg}^{+2}\right)$, potássio disponível $\left(\mathrm{K}^{+}\right)$, fósforo disponível(P), enxofre (S), CTC efetiva (T), soma de bases (SB), saturação de bases (V) e matéria orgânica (MO). Os resultados foram submetidos à analise de variância e as médias, comparadas pelo teste de Scott-Knott. Após a análise dos dados, concluiu-se que, embora tenha havido diferenças entre alguns elementos estudados, as características químicas dos solos nos três sistemas não foram severamente afetadas.
\end{abstract}

Palavras-chave: Agrosilvicultura, Inga vera Willd e Grevílea robusta A. Cunn.

\section{EVALUATION OF SOIL FERTILITY IN AGROFOREST SYSTEMS WITH COFFEE TREES (Coffea arabica L.) IN LAVRAS-MG}

\begin{abstract}
The objective of this wark was to evaluat e the soil fertility in an agroforest system using coffee trees (Coffea arabica L-Mundo Novo), inga trees (Inga vera Wild) and grevillea trees (Grevillea robusta A Cunn) situated in Lavras, Minas Geris. A completely randomized experimental design with tree treatments and seven replicates was utilized. The treatments were : a) coffee trees in full sunshine; $b$ ) coffee trees mixed with inga trees and c) coffee trees mixed with grevillea trees. Tree spacings in the three systems were $4 x$ $1 \mathrm{~m}$ for coffee, $8 \mathrm{~m} \times 15 \mathrm{~m}$ for inga and $12 \times 10 \mathrm{~m}$ for grevillea. With coffee and inga trees at 15 years of age and grevillea trees at 9 years of age, the following soil characteristics were evaluated; $p H$, potential acidity $\left(\mathrm{H}^{+} \mathrm{Al}\right)$, exchangeable aluminum $\left(\mathrm{Al} \mathrm{l}^{+3}\right)$, exchangeable bases $\left(\mathrm{Ca}^{+2}\right.$ and $\left.\mathrm{Mg}^{+2}\right)$, available potassium $\left(K^{+}\right)$, available phosphorus $(P)$, sulfur $(S)$, effective $C E C(t)$, base sum $(B S)$, base saturation $(V)$ and organic matter $(O M)$. The results were subjected to the analysis of variance and means compared by the Scott-Knott test. After data analysis, it was concluded that although there were differences among some studied parameters, the chemical characteristics of the soils under the three systems were not severely affected.
\end{abstract}

Keywords: Agrosilviculture, Inga vera Willd and Grevillea robusta A . Cunn.

\footnotetext{
${ }^{1}$ Recebido em 15.09.2004 e aceito para publicação em 05.04.2006.

${ }^{2}$ DCF, Universidade Federal de Lavras, Lavras-MG.

${ }^{3}$ EPAMIG/CTSM
} 


\section{INTRODUÇÃO}

Entre as diversas técnicas de manejo utilizadas pela agricultura surgiram os sistemas agroflorestais (SAFs), formas de cultivo em que se consorciam, em uma mesma área, espécies arbóreas e cultivos agrícolas e, ou, animais. Os estudos envolvendo tais sistemas têm avançado muito em várias regiões do Brasil, incentivados tanto pelo uso de práticas conservacionistas do solo e da água quanto pela manutenção de produtividades satisfatórias e até mesmo com maiores retornos ao produtor.

O estudo dos SAFs produtivos torna-se de suma importância pela inclusão de uma nova variável a ser entendida, ou seja, a interação/relação que ocorre entre os cultivos agrícolas e as árvores presentes nos sistemas agroflorestais. Questões como a erosão e perdas de solo, o comprometimento da produtividade esperada, a competição entre as espécies consorciadas e suas relações abrem um campo atraente na pesquisa, envolvendo a agricultura e a silvicultura conjuntamente (SALGADO, 2004).

Considerando que o solo é a base para uma agricultura e uma produção florestal sustentável, é necessário adotar práticas de manejo que conservem e, ou, restaurem sua fertilidade, a fim de manter a produtividade (ALVARENGA, 1996). O uso intenso das terras exploradas com culturas perenes ressalta a necessidade de se manter uma exploração racional, a fim de preservar o potencial produtivo dos solos; assim, o conhecimento das propriedades químicas e físicas do solo é uma ferramenta fundamental para direcionar práticas que reduzam o depauperamento a níveis toleráveis (THEODORO, 2001).

A condução convencional das lavouras cafeeiras, formadas em monocultivos, e seus efeitos sobre os ecossistemas têm sido observados em vários estudos. Alvarenga e Souza (1995) citaram que a poluição química dos solos, causada pelo uso indevido de pesticidas e doses excessivas de fertilizantes, pode promover um desbalanço de cargas no ambiente, com uma conseqüente alteração da atividade microbiana e demais conseqüências sobre os aspectos físicos do solo, inclusive poluição e, ou, eutrofização de mananciais.

A lavoura cafeeira, quando indevidamente manejada, pode trazer conseqüências negativas ao solo, como compactação e perda de nutrientes via erosão, podendo trazer reflexos negativos na produção (CUNHA, 1995).
Segundo Malavolta (1993), a origem da acidez dos solos pode ser explicada por quatro processos mais importantes: (a) lixiviação de bases ao longo dos anos (Ca, Mg e, em menor grau, K), que são substituídas por íons $\mathrm{H}^{+}$e, principalmente, íons $\mathrm{Al}^{+3}$, os quais, em parte, passam para a solução do solo; (b) processos de troca de cátions da solução do solo $\left(\mathrm{K}^{+}, \mathrm{Ca}^{+2} \mathrm{e} \mathrm{Mg}^{+2}\right.$, principalmente), durante a absorção radicular, por íons $\mathrm{H}^{+}$; (c) adubos nitrogenados não nítricos, ao serem nitrificados no solo, geram $\mathrm{H}^{+}$; e (d) utilização do cloreto de potássio durante a adubação aumenta os teores de Al e Mn, componentes da acidez do solo.

Inserido nesse contexto, o objetivo deste trabalho foi analisar as alterações ocorridas nas características químicas dos solos de lavouras cafeeiras cultivadas sob sistemas agroflorestais (cafeeiros consorciados com ingazeiros e cafeeiros com grevílea) e convencionalmente a pleno sol.

\section{MATERIAL E MÉTODOS}

O presente trabalho foi desenvolvido em lavouras cafeeiras de propriedade particular no Município de Lavras, sul de Minas Gerais, onde se encontram os cultivos de cafeeiro a pleno sol, cafeeiro consorciado com ingá e cafeeiro consorciado com grevílea, em sistemas agroflorestais.

A cidade de Lavras localiza-se no sul do Estado de Minas Gerais, a $900 \mathrm{~m}$ de altitude, latitude de $21^{\circ} 14^{\prime} \mathrm{S}$ e longitude de $45^{\circ} 00^{\prime} \mathrm{W}$. Apresenta clima do tipo CWa, segundo a classificação de Köppen. A temperatura média anual é de $19,4^{\circ} \mathrm{C}$, com a média da máxima de $26,1^{\circ} \mathrm{C}$ e a média da mínima de $14,8^{\circ} \mathrm{C}$; a precipitação média anual é de $1.529,7 \mathrm{~mm}$ (BRASIL, 1992).

O cafeeiro estudado é da variedade Mundo Novo, plantado no espaçamento 4 x $1 \mathrm{~m}$ nos três sistemas e com mesma idade. Os cafeeiros foram recepados há cinco anos, encontrando-se em plena produtividade, sendo que nos sistemas agroflorestais ocorrem as seguintes distribuições espaciais:

1) Cafeeiro $x$ ingazeiro - Uma linha de cafeeiro arborizada e uma linha solteira, árvores distanciadas a $15 \mathrm{~m}$ na linha de plantio e $8 \mathrm{~m}$ na entrelinha. O ingazeiro foi plantado juntamente com os cafeeiros, estando hoje com 15 anos (Figura 1). 
Cafeeiro x grevílea - Uma linha de cafeeiro arborizada e duas linhas solteiras, árvores distanciadas a $10 \mathrm{~m}$ na linha de plantio e $12 \mathrm{~m}$ na entrelinha. A grevílea foi plantada com o cafeeiro já formado, estando hoje com nove anos (Figura 2).

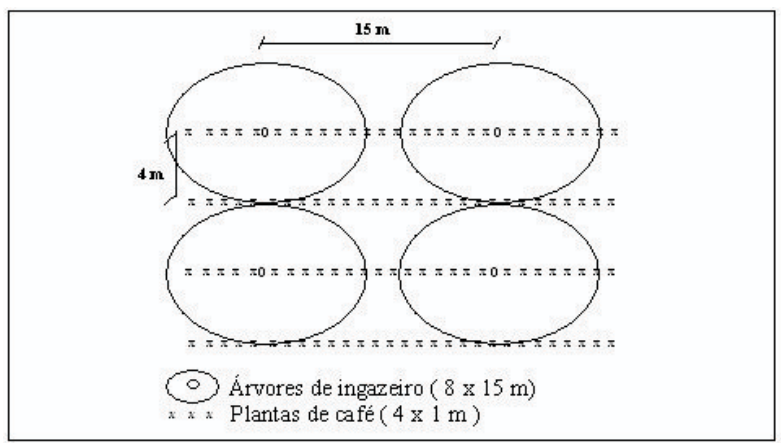

Figura 1 - Representação do cultivo de cafeeiros consorciados com Inga vera Willd.

Figure 1-Representation of the cultivation of coffee trees mixed with Inga vera Willd.

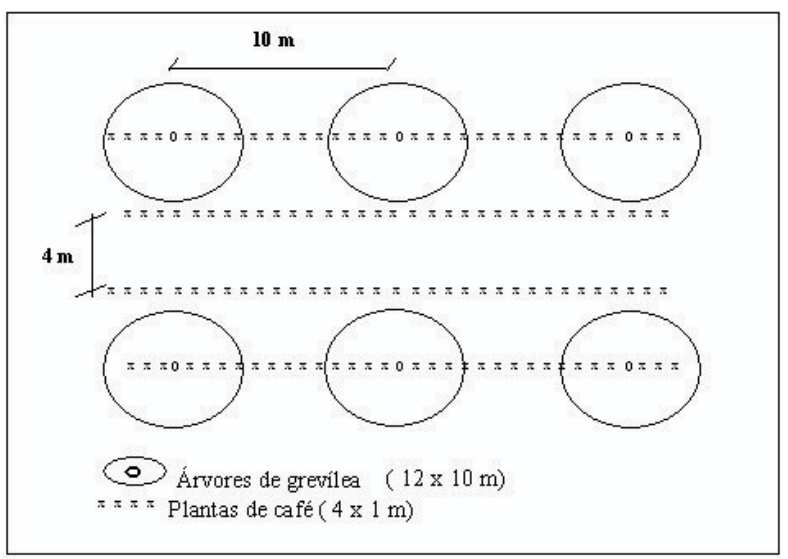

Figura 2 - Representação do cultivo de cafeeiros consorciados com Grevilea robusta A. Cunn.

Figure 2-Representation of the cultivation of coffee trees mixed with Grevillea robusta A. Cunn.

O delineamento experimental utilizado foi o inteiramente casualizado, composto por três tratamentos (cafeeiros x ingazeiros, cafeeiros x grevílea e cafeeiros a pleno sol) e sete repetições. As parcelas onde foram coletadas as amostras de solo para as avaliações edáficas foram compostas por 66 plantas de cafeeiros (três linhas de 22 plantas), sendo consideradas úteis as 20 plantas internas da linha central e as outras como a bordadura.
As amostragens de fertilidade do solo foram realizadas em setembro de 2003, com trado-holandês na projeção da copa do cafeeiro, numa profundidade de $0-20 \mathrm{~cm}$, nas sete repetições, em cada um dos três sistemas de cultivo do cafeeiro estudados. Em cada repetição foram estabelecidos cinco pontos de amostragem nas 20 plantas úteis de cada parcela amostral dos dois lados do cafeeiro. As amostras simples desses cinco pontos foram homogeneizadas, sendo retirada uma amostra de aproximadamente $1,0 \mathrm{~kg}$ de material de solo, que foi colocada em saco plástico e identificada.

As análises químicas foram: $\mathrm{pH}$, acidez potencial $(\mathrm{H}+\mathrm{Al})$, alumínio trocável $\left(\mathrm{Al}^{+3}\right)$, bases trocáveis $\left(\mathrm{Ca}^{+2}\right.$ e $\mathrm{Mg}^{+2}$ ), potássio disponível $\left(\mathrm{K}^{+}\right)$, fósforo disponível (P), enxofre (S), CTC efetiva (t), soma de bases (SB), saturação de bases (V) e matéria orgânica (MO).

As amostras de material de solo foram analisadas no Laboratório de Fertilidade do Solo do Departamento de Ciência do Solo da UFLA, conforme metodologia descrita a seguir: pH em água em relação 1: 2,5 (solo: água), de acordo com o método proposto por Mclean (1982); o alumínio trocável foi extraído com KCl $1 \mathrm{~N}$ e analisado por titulometria com $\mathrm{NaOH} 0,025 \mathrm{~N}$ (BARNHISEL e BERTSCH, 1982); as bases trocáveis foram extraídas com $\mathrm{KCl} 1 \mathrm{~N}$ e determinadas por titulometria com EDTA 0,025 N (LANYON e HEALD, 1982); o potássio disponível foi obtido com solução extratora Mehlich I ( $\left.\mathrm{HCl} 0,05 \mathrm{~N}+\mathrm{H}_{2} \mathrm{SO}_{4} 0,025 \mathrm{~N}\right)$ e analisado por fotometria de chama, e o fósforo disponível foi determinado pelo método da resina (EMBRAPA, 1988). O enxofre foi determinado por turbidimetria (BLANCHAR et al., 1965).

Os resultados obtidos foram submetidos à análise de variância e as médias, comparadas pelo teste de Scott-Knott, e esses resultados foram comparados com os níveis críticos para a cultura do cafeeiro proposto pela CFSEMG (1999).

De modo a caracterizar os componentes arbóreos nos sistemas estudados, em cada parcela amostral foram avaliados os seguintes dados das árvores de ingazeiro e grevílea: altura total, diâmetro à altura do peito (DAP) e área de projeção de copa. A área de projeção de copa (expressa em $\mathrm{m}^{2}$ ) foi obtida tomando-se duas medidas, o comprimento da copa no sentido das linhas de cafeeiro ( P1) e comprimento no sentido perpendicular às linhas de plantio ( P2 ) e calculada através da fórmula utilizada por Macedo (1991):

$$
\text { A área de projeção de copa: } \frac{\mathrm{P} 1 * \mathrm{P} 2 * \pi}{4}
$$

R. Árvore, Viçosa-MG, v.30, n.3, p.343-349, 2006 


\section{RESULTADOS E DISCUSSÃO}

Todos os resultados de análise de solo foram comparados segundo padrões de fertilidade da $5^{\text {a }}$ aproximação, da CFSEMG (1999).

Na Tabela 1 são apresentados o resumo da análise de variância, o coeficiente de variação e a média geral dos macronutrientes $\mathrm{P}, \mathrm{K}, \mathrm{Ca}, \mathrm{Mg}$ e da acidez potencial $(\mathrm{H}+\mathrm{Al}),(\mathrm{pH})$, soma de bases trocáveis $(\mathrm{SB})$, capacidade de troca catiônica efetiva (t), saturação de bases (V) e matéria orgânica (MO).

Foram detectados valores nulos de Al nos solos, provavelmente devido aos valores de $\mathrm{pH}$ observados, reduzindo a solubilidade de Al, e também pela provável reação de complexação de Al com compostos orgânicos (PAVAN, 1983; ALCÂNTARA, 1997).

Em todos os sistemas de cultivo estudados, o maior valor observado para a saturação de alumínio ( $\mathrm{m} \%$ ) foi de $9 \%$, valor bastante inferior ao tolerado pelo cafeeiro (25\%), segundo CFSEMG (1999). Assim, optou-se por não considerar a saturação de alumínio nas comparações realizadas.

Com relação ao $\mathrm{pH}$ dos solos estudados, todos os sistemas apresentaram um padrão de acidez média $(5,1-6,0)$ quanto à classificação química. Quanto à classificação agronômica, o sistema cafeeiro x ingazeiro apresentou um valor de 5,4, considerado "baixo" (4,5 $-5,4)$, enquanto nos outros sistemas o nível foi considerado "bom" $(5,5-6,0)$.
Pela Tabela 2, nota-se que o pH do solo não mostrou diferença significativa entre os sistemas avaliados, sendo que os nutrientes $\mathrm{P}, \mathrm{K}$ e Mg também mostraram a mesma igualdade, com exceção do Ca, para o qual foi observado maior valor no sistema a pleno sol. A acidez potencial $(\mathrm{H}+\mathrm{Al})$ e a de saturação por bases (V) não mostraram diferença significativa entre os sistemas, já a soma de bases (SB), a matéria orgânica (MO) e a CTC efetiva (t) mostraram-se com maiores valores nos solos em que os cafeeiros são cultivados a pleno sol.

Os teores de matéria orgânica nos três sistemas de cultivo foram considerados nível "médio" ( 2,10 a 4,0 dag/kg) e a CTC efetiva nível "médio", para os SAFs (2,31 a 4,6 cmol $\left./ \mathrm{dm}^{3}\right)$ e nível "bom" (4,61 a 8,0 $\mathrm{cmol}_{\mathrm{c}} / \mathrm{dm}^{3}$ ) para o cultivo a pleno sol.

Maiores valores observados para matéria orgânica e pH nos sistemas a pleno sol podem ter afetado diretamente a disponibilidade de $\mathrm{P}$, que, apesar de não apresentar diferença entre os sistemas, atingiu maiores valores no sistema a pleno sol. Segundo Fernandes et al. (1997), ocorre maior quantidade de $\mathrm{P}$ nas camadas superficiais do solo, correlacionado com maior teor de matéria orgânica.

Os cafeeiros a pleno sol perdem mais folhas que os sombreados, uma vez que o microclima a pleno sol é mais seco e, assim, precisam reduzir a respiração no período de seca, derrubando suas folhas no solo (MOREIRA, 2003).

Tabela 1 - Resumo das análises de variância, coeficientes de variação e médias gerais para os nutrientes $\mathrm{P}, \mathrm{K}, \mathrm{Ca}$ e Mg, $\mathrm{pH}$, acidez potencial $(\mathrm{H}+\mathrm{Al})$, soma de bases trocáveis $(\mathrm{SB})$, capacidade de troca catiônica efetiva $(\mathrm{t})$, índice de saturação de bases (V) e matéria orgânica (MO) encontrados em análise de solo de cafeeiros cultivados em diferentes sistemas de cultivo

Table 1 -Summary of the analysis of variance, coefficients of variation and general means for the nutrients $P, K$, Ca and $M g, p H$, potential acidity $(H+A l)$, exchangeable base summation $(S B)$, effective cation exchange capacity $(t)$, base saturation index $(V)$ and organic matter $(O M)$ found in analysis of soil under coffee trees grown in different cultivation systems

\begin{tabular}{|c|c|c|c|c|c|c|c|c|c|c|c|}
\hline \multirow[t]{2}{*}{$\mathrm{FV}$} & \multirow[t]{2}{*}{ GL } & \multicolumn{10}{|c|}{ Quadrado Médio } \\
\hline & & $\mathrm{pH}$ & $\begin{array}{c}\mathrm{P} \\
\mathrm{mg} / \mathrm{dm}^{3}\end{array}$ & $\begin{array}{c}\mathrm{K} \\
\mathrm{mg} / \mathrm{dm}^{3}\end{array}$ & $\begin{array}{c}\mathrm{Ca} \\
\mathrm{cmol}_{\mathrm{c}} / \mathrm{dm}^{3}\end{array}$ & $\begin{array}{c}\mathrm{Mg} \\
\mathrm{cmol} / \mathrm{dm}^{3}\end{array}$ & $\begin{array}{c}\mathrm{H}+\mathrm{Al} \\
\mathrm{cmol} / \mathrm{dm}^{3}\end{array}$ & $\begin{array}{c}\mathrm{SB} \\
\mathrm{cmol}_{\mathrm{c}} / \mathrm{dm}^{3}\end{array}$ & $\begin{array}{c}\mathrm{t} \\
\mathrm{cmol} / \mathrm{dm}^{3}\end{array}$ & $\begin{array}{c}\mathrm{MO} \\
\mathrm{dag} / \mathrm{kg}\end{array}$ & $\begin{array}{l}\mathrm{V} \\
\% \\
\end{array}$ \\
\hline Trat & 2 & $0,206 \mathrm{~ns}$ & $58,30 \mathrm{~ns}$ & $433,8 \mathrm{~ns}$ & $4,98 * *$ & $0,043 \mathrm{~ns}$ & $0,798 \mathrm{~ns}$ & $6,66^{* *}$ & $6,19 * *$ & $1,49 * *$ & $90,36 \mathrm{~ns}$ \\
\hline Erro & 18 & 0,13 & 60,49 & 280,6 & 0,36 & 0,046 & 1,55 & 0,45 & 0,41 & 0,05 & 88,40 \\
\hline \multicolumn{2}{|c|}{$\overline{\mathrm{CV}(\%)}$} & 6,57 & 35,76 & 16,10 & 22,04 & 24,93 & 28,61 & 17,52 & 16,44 & 9,77 & 19,78 \\
\hline \multicolumn{2}{|c|}{ Média geral } & 5,58 & 21,74 & 104,0 & 2,73 & 0,86 & 4,35 & 3,84 & 3,90 & 2,45 & 47,53 \\
\hline
\end{tabular}

** Significativo a $1 \%$ de probabilidade e $\mathrm{ns}=$ não-significativo.

R. Árvore, Viçosa-MG, v.30, n.3, p.343-349, 2006 
Tabela 2 - Valores médios dos nutrientes $\mathrm{P}, \mathrm{K}, \mathrm{Ca}$ e Mg, pH, acidez potencial (H+Al), soma de bases trocáveis (SB), capacidade de troca catiônica efetiva (t), índice de saturação de bases $(V)$ e matéria orgânica $(\mathrm{MO})$ encontrados em análise de solo de cafeeiros cultivados em diferentes sistemas de cultivo

Table 2 - Mean values for the nutrients $P, K, C a$ and $M g, p H$, potential acidity $(H+A l)$, exchangeable base summation $(S B)$, effective cation exchange capacity $(t)$, base saturation index $(V)$ and organic matter $(O M)$ found in analysis of soil under coffee trees grown in different cultivation systems

\begin{tabular}{|c|c|c|c|c|c|c|c|c|c|c|}
\hline Sistema & $\mathrm{pH}$ & $\begin{array}{c}\mathrm{P} \\
\mathrm{mg} / \mathrm{dm}^{3}\end{array}$ & $\begin{array}{c}\mathrm{K} \\
\mathrm{mg} / \mathrm{dm}^{3}\end{array}$ & $\begin{array}{c}\mathrm{Ca} \\
\mathrm{cmol}_{\mathrm{c}} / \mathrm{dm}^{3}\end{array}$ & $\begin{array}{c}\mathrm{Mg} \\
\mathrm{cmol}_{\mathrm{c}} / \mathrm{dm}^{3}\end{array}$ & $\begin{array}{c}\mathrm{H}+\mathrm{Al} \\
\mathrm{cmol} / \mathrm{dm}^{3}\end{array}$ & $\begin{array}{c}\mathrm{SB} \\
\mathrm{cmol}_{\mathrm{c}} / \mathrm{dm}^{3}\end{array}$ & $\begin{array}{c}\mathrm{t} \\
\mathrm{cmol} / \mathrm{dm}^{3}\end{array}$ & $\begin{array}{c}\mathrm{MO} \\
\mathrm{dag} / \mathrm{kg}\end{array}$ & $\begin{array}{l}\mathrm{V} \\
\% \\
\end{array}$ \\
\hline (I) & $5,4 \mathrm{a}$ & $21,7 \mathrm{a}$ & $96,4 \mathrm{a}$ & $2,0 \mathrm{~b}$ & $0,81 \mathrm{a}$ & $4,11 \mathrm{a}$ & $3,00 \mathrm{~b}$ & $3,0 \mathrm{~b}$ & $2,2 b$ & $43,8 \mathrm{a}$ \\
\hline (G) & $5,6 a$ & $18,8 \mathrm{a}$ & $103, a$ & $2,1 b$ & $0,82 \mathrm{a}$ & $4,21 \mathrm{a}$ & $3,62 b$ & $3,7 b$ & $2,1 b$ & $47,6 \mathrm{a}$ \\
\hline (P) & $5,7 \mathrm{a}$ & $24,6 \mathrm{a}$ & $112, \mathrm{a}$ & $3,6 \mathrm{a}$ & $0,95 \mathrm{a}$ & $4,74 \mathrm{a}$ & $4,91 \mathrm{a}$ & $4,91 \mathrm{a}$ & $2,9 a$ & $51,0 \mathrm{a}$ \\
\hline
\end{tabular}

Valores precedidos de mesma letra na coluna não diferem, significativamente, a 5\% de probabilidade, pelo teste de Scott-Knott. (I) cafeeiros $\mathrm{x}$ ingazeiros, $(\mathrm{G})$ cafeeiros $\mathrm{x}$ grevílea e (P) cafeeiros a pleno sol.

Fernandes (1986) e Matiello et al. (2002), citados por Moreira (2003), relataram que no sistema a pleno sol existe maior número de espécies espontâneas nas entrelinhas do café, gerando maior biomassa. Já, no cafezal sombreado, as espécies espontâneas, principalmente as gramíneas, são inibidas. Assim, a grande quantidade de "mulch" presente nos sistemas agroflorestais atuaria impedindo o crescimento de espécies invasoras, e o maior acúmulo de matéria orgânica advinda da capina dessas plantas poderia também estar explicando maiores valores de matéria orgânica nos cultivos a pleno sol.

A característica soma de bases (SB) foi classificada de "médio" (1,81 a 3,6 cmol $\left./ \mathrm{dm}^{3}\right)$ para o sistema cafeeiro x ingazeiro e "bom" $\left(3,61 \mathrm{a} 6,0 \mathrm{cmol} / \mathrm{dm}^{3}\right)$ para os demais sistemas, conforme CFSEMG (1999). A característica acidez potencial $(\mathrm{H}+\mathrm{Al})$ foi classificada como “médio"(2,51 a 5,0 $\left.\mathrm{cmol}_{\mathrm{c}} / \mathrm{dm}^{3}\right)$, não apresentando diferenças entre os três sistemas.

O teor de potássio no solo foi classificado como "médio" (60 a 120 mg/dm³ ), não diferindo para os diferentes sistemas de cultivo. Os teores de cálcio trocável foram considerados como "bom" $\left(2,41\right.$ a $\left.4,0 \mathrm{cmol}_{\mathrm{c}} / \mathrm{dm}^{3}\right)$ para os cultivos a pleno sol e "médio" $(1,21$ a $2,4 \mathrm{cmol}$ / $\mathrm{dm}^{3}$ ) para os solos avaliados nos sistemas agroflorestais. O magnésio seguiu a mesma tendência do cálcio, apresentando nível "bom" $\left(0,91\right.$ a $\left.1,5 \mathrm{cmol}_{\mathrm{c}} / \mathrm{dm}^{3}\right)$ para os cultivos a pleno sol e "médio" $(0,46$ a 0,9 cmol / $\mathrm{dm}^{3}$ ) para os solos avaliados nos sistemas agroflorestais.

Maiores valores encontrados nos solos para as variáveis $\mathrm{Ca}, \mathrm{SB}, \mathrm{MO}$ e (t), nos cultivos a pleno sol, provavelmente devem-se a um efeito competitivo entre as árvores e os cafeeiros nos sistemas agroflorestais, em que a competição exercida pelas árvores estaria promovendo uma diminuição nos nutrientes do solo, principalmente o cálcio, e outros que teriam influência na composição das variáveis bases trocáveis e capacidade troca catiônica efetiva.

Nos ecossistemas florestais, o fluxo de elementos minerais forma um ciclo entre o dossel florestal e o solo, o que explica o crescimento vegetal exuberante sobre solos pobres (ALVARENGA, 1996). Segundo esse autor, o mecanismo de ciclagem forma um ciclo fechado, em que os nutrientes são transferidos para o solo, principalmente pela queda de folhas e outras partes da planta, onde são decompostos e lixiviados pela água das chuvas, chegando ao alcance das raízes para serem novamente absorvidos.

Espera-se que essa menor fertilidade dos solos no sistema consorciado com ingazeiro se deve à alta densidade de árvores e ao acúmulo de nutrientes em sua parte aérea e raízes, apresentando maior similaridade com as condições supracitadas de um ecossistema natural do que com os monocultivos e até mesmo os cultivos com a espécie exótica (grevílea).

Theodoro (2001), que comparou os efeitos do manejo em cafeeiro orgânico, convencional e em conversão, constatou que houve incrementos no $\mathrm{pH}$ e nos valores de Ca, Mg, K, P, S, Zn, B, CTC do solo, V(\%), diminuindo ainda o Al trocável. Esses efeitos foram maiores nos cafeeiros orgânicos, seguidos pelo cafeeiro em conversão. Segundo essa autora, esses maiores incrementos estão diretamente relacionados com a prática da adubação orgânica e cobertura vegetal permanente do solo.

Apesar de haver constante cobertura do solo nos sistemas agroflorestais, com folhas e galhos das árvores, essas características foram insuficientes para afetar

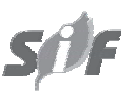

R. Árvore, Viçosa-MG, v.30, n.3, p.337-341, 2006 
o teor de matéria orgânica do solo nos sistemas com cafeeiros arborizados. Durante as coletas de solo para análise, foi observado grande quantidade de raízes do cafeeiro presente diretamente nesse manto formado pelas folhas, acima do solo, o que pode explicar, em parte, a menor presença de matéria orgânica incorporada ao substrato. Pois esse manto superficial é separado da porção de solo que será enviada para análise da matéria orgânica, portanto não é considerada nos valores finais desta pesquisa.

As árvores de ingazeiro não apresentam sombreamento fechado, devido à disposição de seus ramos ascendentes e irregulares e à sua copa ampla, com crescimento horizontal e parcialmente desfolhada. A altura média dos ingazeiros foi de $12,6 \mathrm{~m}$ e o DAP médio, de 77,3 cm por árvore. A área de projeção de copa média foi de $176,2 \mathrm{~m}^{2}$, com as árvores bifurcando a uma altura média de $0,93 \mathrm{~cm}$, sendo que em alguns indivíduos a bifurcação iniciava-se à altura do solo.

Nas árvores de grevílea componentes do SAF, a altura média foi de 8,7 m e o DAP médio, de 23,9 $\mathrm{cm}$ por árvore, sendo a área de projeção de copa média de $26 \mathrm{~m}^{2}$. Vale destacar que a grevílea apresenta fuste reto, ideal para a utilização de madeira serrada.

Os componentes arbóreos presentes na lavoura cafeeira, além de favorecerem incrementos na produtividade dos cafeeiros, mantêm relação ecológica altamente positiva com esses ambientes, aumentando a biodiversidade e favorecendo a presença de espécies que normalmente não ocorrem nos monocultivos de cafeeiro. Durante o período de coleta dos dados, pôdese observar a presença constante de fauna silvestre na área arborizada, principalmente de indivíduos da avifauna, que utilizam as árvores para alimentação, descanso e nidificação.

\section{CONCLUSÕES}

- O solo nos cultivos a pleno sol apresentaram maiores valores para matéria orgânica (MO), CTC efetiva ( t ), soma de bases (SB) e teores de Ca em comparação com os SAFs.

- As características $\mathrm{pH}$, acidez potencial $(\mathrm{H}+\mathrm{Al})$, saturação de bases (V) e os teores de alumínio, magnésio, potássio, fósforo e enxofre não apresentaram diferença estatística entre os diferentes sistemas de cultivo do cafeeiro.

R. Árvore, Viçosa-MG, v.30, n.3, p.337-341, 2006
- Condições químicas dos solos apresentaramse, no geral, com melhores características nos monocultivos, o que pode ter ocorrido devido à demanda nutricional das árvores.

- Maiores espaçamentos entre as árvores poderiam minimizar as diferenças de fertilidade observadas entre os sistemas arborizados e os cultivos a pleno sol do cafeeiro.

\section{REFERÊNCIAS BIBLIOGRÁFICAS}

\author{
ALCANTARA, E. N. Efeito de diferentes \\ métodos de controle de plantas \\ daninhas na cultura do cafeeiro (Coffea \\ arabica L.) sobre a qualidade de um \\ Latossolo Roxo Distrófico. 1997. $133 f$. \\ Tese (Doutorado em Fitotecnia) - Universidade \\ Federal de Lavras, Lavras, 1997.
}

ALVARENGA, M. I. N. Propriedades físicas, químicas e biológicas de um Latossolo Vermelho-Escuro em diferentes ecossistemas. 1996. 211f. Tese (Doutorado em Fitotecnia) Universidade Federal de Lavras, Lavras, 1996.

ALVAREnGA, M. I. N.; SOUZA, J. A. Atributos do solo e o impacto ambiental. Lavras: Escola Superor de Lavras, 1995. 140 p.

(Especialização - Tutoria à distância em Solos e Meio Ambiente).

BARNHISEL, R.; BERTSHCH, P. M. Aluminium. In: PAGE, A. L.; MILLER, R. H.; KEENEY, D. R. Methods of soil analysis: chemical and microbiological properties. 2. ed. Madison: America Society of Agronomy, 1982. Pt. 2. p. 275-296.

BLANCHAR, R. W.; REHM, G.; CALDWELL, A. C. Sulfur in plant material digestion with nutric and percloria acid. Soil Science Society Proceedings, v. 29, n. 1, p.71, 1965.

BRASIL. Ministério da Agricultura e Reforma Agrária. Normais climatológicas de 1961- 1990. Brasília: 1992, 84 p.
COMISSÃO DE FERTILIDADE DO SOLO DO ESTADO DE MINAS GERAIS - CFSEMG.
Recomendações para o uso de corretivos e fertilizantes em Minas

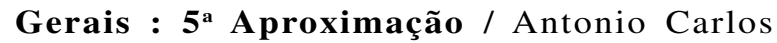 Ribeiro, Paulo Tácito Gontijo Guimarães, Vitor Hugo Alvarez V.( Eds.) Viçosa, MG: 1999. 359p. 
CUNHA, G. M. Estudo comparativo de condições químicas e físicas de um latossolo Vermelho-Amarelo Álico, de encosta, sob duas coberturas: café e mata natural. 1995. 65f. Dissertação (Mestrado em Fitotecnia) - Universidade Federal de Viçosa, Viçosa, 1995.

EMPRESA BRASILEIRA DE PESQUISA AGROPECUÁRIA - EMBRAPA. Serviço Nacional de Levantamento e Conservação de Solos.

Manual de métodos de análise de solo. Rio de Janeiro: 1988. 67 p. (Documento, 11).

FERNANDES, L. A. et al. Propriedades químicas e bioquímicas de solos sob vegetação de mata e campos cerrado adjacentes. Ciência e Agrotecnologia, v. 21, n. 1, p. 58-70, 1997.

LANYON, L. E.; HEALD, W. R. Magnesium, calcium and barium. In: PAGE, A. L.; MILLER, R. H.; KEENEY, D. R. Methods of soil analysis: chemical and microbiological properties. 2. ed. Madison: ASA, 1982. Pt. 2, p. 247-260.

MACEDO, R. L. G.; Avaliação Holística da Fase Juvenil do Teste de Introdução de Espécies de Eucalyptus na Baixada Cuiabana, Mato Grosso. 1991. 231f. Tese (Doutorado em Engenharia Florestal) Universidade Federal do Paraná, Curitiba, 1991.

MALAVOLTA, E. Nutrição mineral e adubação do cafeeiro, colheitas máximas econômicas. São Paulo: Agronômica Ceres, 1993. 210 p.
McLEAN, E.O. Soil pH and lime requirement. In: PAGE, A.L.; MILLER, R.H.; KEENEY, D.R.

Methods of soil analysis: chemical and microbiological properties. 2.ed. Madison:ASA, 1982. Pt.2, p.199-223.

MOREIRA, C. F. Caracterização de sistemas de café orgânico sombreado e a pleno sol no sul de Minas Gerais. 2003. 78f. Dissertação (Mestrado em Ecologia de Agroecossistemas) - Escola Superior de Agronomia Luiz Queiroz, Piracicaba, 2003.

PAVAN, M. A. Alumínio em solos ácidos do Paraná: relação entre alumínio não-trocável, trocável e solúvel com $\mathrm{pH}, \mathrm{DTC}$, porcentagem de saturação de Al e matéria orgânica. Revista Brasileira de Ciência do Solo, v. 7, n. 1, p. 39-47, 1983.

SALGADO, B.G. Caracterização de sistemas agroflorestais com cafeeiro em Lavras - MG. 2004. 115f. Dissertação (Mestrado em Engenheria Florestal) -

Universidade Federal de Lavras, Lavras, 2004.

THEODORO, V. C. A. Caracterização de sistemas de produção de café orgânico, em conversão e convencional. 1999. 214f. Dissertação (Mestrado em Fitotecnia) - Universidade Federal de Lavras, Lavras, 1999. 